\title{
Special issue on the abuse and misuse of social agents
}

\begin{abstract}
"You see, there's a primal joy in hitting a thing in motion. It's one of the oldest pleasures there is. Something moves, boo, you wing it. Beast, bird or human, the thing to do is to knock it down. It's primal, Davy. It's basic to the origin of the species."
\end{abstract}

- Don DeLillo, Americana

\section{Introduction}

Coming across an unknown creature generates anxiety. Its autonomy gives rise to fears; its agency requires vigilance. In the primitive world of predators and prey, it is wise to be leery of anything that moves and to learn as quickly as possible whether a creature is aggressive or possesses dangerous weapons of self-defence. What better way to find that out then to throw a rock at it from a safe distance to see how it reacts and what it is capable of doing to protect itself. Testing, teasing, and poking creatures is so crucial for human and animal survival that it is no wonder these behaviours are also pleasurable, as are play fighting and play chasing (for a review of the literature, see Aldis, 1975). Apparently an elementary predator-prey schema runs across cultures and most species (Lorenz, 1939).

Novel mechanical inventions, such as the automobile, can trigger the same predator-prey schema. In the early 20th century, for example, it was not uncommon for people to behave towards cars and tractors as they would towards unknown animals. As reported in Billerter (1997), a groom once reacted to the klaxon of a car going off as he would to the growl of a wild animal, warning his master, "Sir, sir, don't go in there, the car is gone crazy, it might take the bit between its teeth and run you over." (p. 1).

Ongoing familiarity with machines quickly corrects mistaken human and animal attributions and primitive fears, but in recent years interactive machines have been developed that look like human beings and that talk and imitate human behaviour. These machines are designed to elicit the natural tendency to animate and to anthropomorphize. Developers would love nothing more than to create beings that sustain the suspension of disbelief for the long term in a real world environment. A motivating force behind this technology is the goal of creating virtual collaborators, partners, and assistants. As a result, a pervasive trend in interaction design is the attempt of finding ways to establish viable social relationships between machines and their users. Anthropomorphic cues and varied levels of technological sophistication have been employed in this challenge. Embodied conversational agents (ECA) (Cassell, 2000) are a favourite interface solution in many computer-supported tasks, and relational agents (Bickmore and Picard, 2005) have been proposed to maintain long-term relationships with their users, managing past histories and future expectations. Robotics is moving from industry to people's homes; not only (and not always) do these new robots need to perform useful tasks; but they also need to engage users in such a way that they want to interact with these social machines and care about them.

\subsection{Social agents}

In this special issue, we use the broad term social agent to encompass any form of technological artefact that can be used to establish social relationships with users, independently of the agent's nature (robots and computer interfaces), technological advancement (relational agents and toys), and visual appearance (human-like 3D models and textual interfaces). This definition is rooted in a user-centred perspective; what matters is the user's behaviour and not the nature of the artefact. Social agents provoke anthropomorphic attributions and induce the user to perceive the machine as a social stimulus rather than as a passive object.

Social stimuli are more complex than physical stimuli in that they are causal agents, affecting their partners' behaviour by their presence and through direct interaction (Fiske and Taylor, 1991). Social stimuli are animated; they perceive while they are perceived, change while inducing changes in others, have and elicit intents, motives, desires, and emotions. Furthermore, social stimuli subsume many attributes that cannot be directly observed, such as personality traits, intentions, and desires. These attributes, 
however, need to be inferred and interpreted based on the observer's specific knowledge, expectations, and skills.

Transforming machines from physical objects into social agents is a challenging task that may never be completely achieved. Yet there is much commercial and scientific evidence that virtual relationships, or meaningful encounters between humans and machines, are possible and can become quite important to people. The extremely simple, but hugely popular, Tamagotchi (literally, lovable egg), demonstrated a decade ago that humans can care for machines. Since then, a number of interactive robotic toys have reached the market, often leading to large profits for their creators.

The highly influential media equation theory (Reeves and Nass, 1996), encouraging in its claim that anthropomorphism is inherent to computers, has motivated a wide range of diverse research on social agents (for a meta-analysis, see Yee et al., 2007). In general, this research is characterised by a strong positivistic approach, whereby a range of positive and desirable consequences of social agents are stressed. A preponderance of research examines harmonic encounters and the facilitation effects that the technological medium has on social interactions.

Early evidence of possible negative outcomes of social agents on the user's psychological well-being emerged quite early in the ECA debate, but they were explained in a positive fashion (Rickenberg and Reeves, 2000). In particular, the finding that embodied agents induced a higher level of anxiety in the user (especially when these agents monitored task execution), was explained by associating anxiety with arousal. It was claimed that a higher level of arousal is often desirable in human-computer interaction, since it fosters user attention and responsiveness. As the authors write, "Using an animated character turns up the volume on social presence, which means that it can accentuate the effects of everything presented." (p. 55).

The intention of this special issue is to explore this amplification effect by looking at a larger picture of human-agent interaction, one which also takes into consideration conflicts and the possibility of disinhibited and antisocial behaviour. Expanding the interaction sphere with social agents to encompass emotions and social connections may well lead to constructing virtual antagonists, as well as virtual companions. Social relations are rarely stable but often involve anticipations of disruption and aggression. Many social practices, for example, smiling and apologizing, are in place to stabilise uncertain situations, to appease threats of aggression, and to circumvent harmful emotional eruptions. Yet, all too often, people fight, insult, and betray each other. Why would this dark side of the social disappear when dealing with social agents? In fact, given that social agents, by their talking and exhibition of social cues, are novel machines that are even more animated than automobiles, why would we not expect social agents to trigger more of a need to test the agents out - to poke them and to tease them?

\subsection{Abuse}

We recognize that the title of this special issue, Abuse and Misuse of Social Agents, may annoy or disappoint some readers because of its metaphorical implications. Clearly, the word abuse, when defined as the intent to cause suffering, cannot be applied to inanimate objects, as they are incapable of recognizing, experiencing, or understanding suffering. In Turkle (1997), this point is sharply brought home in the following user's reflections on interacting with Eliza, an artificial therapist:

How could the computer ever, ever have a clue... about what it is like to have your father come home drunk and beat the shit out of you? To understand what was going on here you would need to know what it feels like to be black and blue and know that it's your own father who is doing it to you. (p. 111)

Although we agree with the above statement, we do believe that the metaphor of social agency by itself tends to promote technology to a level that cannot escape the possibility of abuse. If it makes sense to say users are being polite in their responses to a conversational agent when obeying the social rules of politeness, then why would it not make equal sense to say a user is being abusive in his or her responses when using language that, if applied to people, would be considered abusive? Several papers included in this issue show that verbal abuses (e.g., insults, threats, foul language, sexual advances, and pornographic sex-talk) abound in user interactions with conversational agents. Our intention is to openly examine this phenomenon with an eye towards learning what underlying factors provoke abusive behaviour towards social agents. We are also interested in considering the repercussions and the practical and ethical implications of such abuse. We acknowledge the evocative nature of the term abuse, but using this word is consistent with the prevailing anthropomorphic trend current in $\mathrm{HCI}$ discourse.

\subsection{Misuse}

There is another sense of abuse that is of interest to us as well, and that is the misuse, or the inappropriate or unintended uses, of social agents. Our consideration of the misuse of social agents grounds our special issue in design noir (Dunne and Rabby, 2001), an approach to technology that looks at the full spectrum of its use. This perspective portrays the user as a creator of unexpected meanings and applications that can diverge widely from the original intentions of the designer. Users often appropriate technology, misusing it to fit unpredicted needs and desires, and deriving considerable enjoyment from this deviation of intended purposes and procedures. 
Destructive misuses of technology include cyberbullying, electronic spam, and frauds. Although it is impossible to predict all the ways a novel technology will be used, we ask the same question Blay Whitby asks in this issue, and that is, why is it that designers do not spend more time predicting antisocial uses so that they can account for these potential misuses in the original designs of the technology. Design noir provides a framework that stimulates reflections on the less desirable behaviour a product may engender. With very few exceptions (Foucault et al., 2007; Nathan et al., 2007), this framework has had little influence in HCI. The focus in this special issue on misuse intends to extend the design noir perspective to social agents, as their social nature adds to the complexity of the problem.

\section{Contents}

Selecting the papers for this special issue has not been easy, as we received a number of submissions that looked at the phenomenon of abuse and misuse from different perspectives. We decided in this issue to give priority to empirical papers. In addition, we also include an interesting debate on the ethics of abusing robots.

\subsection{Empirical studies}

The empirical section deals with three very different research settings with respect to the interaction context: the technology, the user, and the method. At one extreme, Veletsianos, Scharber, and Doering report the analysis of anonymous conversations between a sample of teenagers and a pedagogical agent collected in a school environment. De Angeli and Brahnam report the analysis of an online corpus of conversation with a chatterbot designed mainly for entertainment and companionship. Mathias Rehm presents a laboratory evaluation of a game where two human beings and an embodied conversational agent participate in a game requiring deception. It is evident that the social acceptability of disinhibition tends to increase in these studies.

Results from the first two studies depict a very similar trend showing that verbal abuse and sexual conversations are common elements of anonymous interaction with conversational agents. The intended focus of the dialogue (learning vs. fun), the interaction setting (school vs. home), and the interface (text only chatterbot vs. embodied agent) appears to have little effect on the occurrence of verbal abuse, which seems to account for some $10 \%$ of the communication (operationalised as dialogue turns in the first study and stem units in the second study). Independent of the context, people appear to enjoy provoking, teasing, and often humiliating the conversational partners. The studies also show an example of a creative misuse of technology. Some users appropriate the conversational agent, transforming it into a toy for practicing proposing sex. This toy seems to be conducive towards disinhibited behaviour, as it does escape traditional moral constraint.
In Rehm's paper, the participants (two humans and an agent) were invited to play a dice game. The objective is to cheat each other. In this evaluation the bandwidth of the interaction is enlarged to encompass nonverbal communication, and the study provides a direct comparison between the behaviour towards the agent and that towards other human beings. Results showed interesting similarities and interesting differences in both gaze behaviour and verbal behaviour. In particular, the agent was looked at more often and for longer periods than was the other person, as if the user were staring at it. Furthermore, the agent was often referred to using the third person, as if it were not present in the interaction. Rehm notes that this is a communication style typically reserved for young children and animals.

\subsection{Ethical reflections}

Although the main aim of this issue is to present some of the first empirical papers on the abuse and misuse of social agents, we also include a full paper by Blay Whitby entitled Sometimes it's hard to be a robot: A call for action on the ethics of abusing artificial agents. This paper calls for serious debate of the ethical issues associated with the human abuse of robots, and, by extension, social agents. Included in this section are invited responses from Alan Dix and Harold Thimbleby.

Whitby begins his ethical call to action by distinguishing social robots from other artefacts, such as cars. Since social robots are designed to emulate human social behaviours, he argues that designers need seriously to ask themselves three new questions. First, is it acceptable for human beings to abuse human-like artefacts in ways that would be considered unacceptable if the robots were people? Second, if it is acceptable to abuse social robots in these ways, then how much abuse should be permitted? Third, is it ethical to design robots to be forgiving of user abuses, as is the current trend? Whitby believes there is an urgent need to address these questions in professional codes of conduct, such as those of the British Computer Society (BCS) and the Association for Computing Machinery (ACM). The paper attempts to lay the ground for exploring these issues.

Thimbleby believes that there is no pressing need to change current professional codes of conduct for the simple reason that robotics fails to extend the range of social consequences, at least not to the degree that merits special consideration. He points out that there are multiple moral layers behind the abuse of robots. On the one hand, certain forms of potential abuse, robot wars, for example, may be instrumental in spurring development in the field. If this is true, then designing robots that rip each other apart provides utilitarian value, and thus should probably be considered good. On the other hand, there is the issue of abuse in terms of inflicting some degree of harm and suffering. Thimbleby looks at this question from both ends of the spectrum: abusing human beings as if they were machines 
(e.g., aborting a foetus that is predicted to be cognitively defective) and abusing machines as if they were human beings. The importance of the latter seems to fade into sentimentalism as Thimbleby broadens and situates Whitby's ethical discussion by looking at critical environmental abuses (those, for example, that lead to global warming) and industrial, personal, and economical abuses of technology (for example, the national financial consequences of not wearing seat belts).

Dix expands Whitby's discussion by pointing out the complexity of the ethical issues. Just as it is wrong for designers to assume that human beings will react to social agents as they do to other interfaces, so it is dangerous to assume "a continuity in ethical implications." Dix begins his ethical discussion by taking a Christian perspective. Rather than focusing on the consequences of human beings abusing social agents, he considers first the social benefits of extending the golden rule to encompass human-like artefacts. An example of the good that can come from this is children learning to be nurturing and kind by tending to the needs of artificial pets. If enhancing positive qualities appeals to our moral sense, then Dix argues that agents that encourage negative behaviours will likewise harm our moral sense. Thus, it would be good for designers to design out unsavoury user behaviours.

Dix goes on to make yet a further claim, and that is that from a Judeo-Christian perspective what distinguishes human beings from other created beings is the fact that human beings are loved. What then happens when people come to love social agents? If social agents are sufficiently human-like to be abused in human-like ways, then is that not reason enough for considering such abuses wrong? And will harming these agents not be similar in moral effect to harming people that are loved?

\section{Concluding remarks}

The idea behind this special issue has evolved in the last few years. The conversation began as a result of informal discussions held at various workshops on conversational agents. Both the authors, along with Peter Wallis, had been doing research on conversational agents for a number of years and were beginning to leave the laboratory to analyse in situ interaction logs, and all of us were struck by the preponderance of verbal abuse discovered in the logs. We were uncertain how to approach this material and began searching the reference literature for guidance. We were surprise to discover that this phenomenon was rarely reported. We finally came to the conclusion that there was a need to address this overlooked material. As a result, we organized two workshops on the subject, one at Interact 2005 entitled, Abuse: The darker side of human computer interaction (De Angeli et al., 2005), and the other, which included Alan Dix, at CHI 2006 entitled, Abuse and misuse of interactive technologies (De Angeli et al., 2006).

These workshops attracted an interdisciplinary audience, producing an interesting cross-fertilization of ideas.
Scholars working in the humanities and in cultural studies greeted the topic with few reservations. Initially, the HCI and ECA communities were underrepresented. Most of the contributions (available at http://www.agentabuse.org) reflected the early stage of the field in HCI and were mostly position papers that addressed possible reasons for and consequences of virtual abuse. Little empirical data on the phenomenon were reported. This special issue reports pioneer empirical studies on how people abuse and misuse conversational technologies. The contributions in this special issue are important, as understanding the needs that motivate negative as well as positive expressions from users will help us build social agents that work and function more constructively within our society.

We hope that this collection of papers will foster reflection and research into this area, inviting the HCI community to take a more balanced approach to the design and evaluation of social agents.

\section{Acknowledgments}

We wish to acknowledge Peter Wallis, Alan Dix, and all the participants in our previous workshops for their contributions in defining this research niche. We also wish to extend our gratitude to the people who helped in the review process for this special issue, for without their expertise this special issue would not have been possible: Hamda Al-Shihi, Christoph Bartneck, Pamela Briggs, Thomas Cavanagh, Lynne Coventry, Chris Creed, Alan Dix, Kerstin Fischer, Linda Weiser Friedman, Bernard Geoghegan, Petra Gieselmann, Peter Goldie, Brigitte Krenn, Sri Kurniawan, Stacey Marsella, Catherine Pelachaud, Zsofia Ruttkay, Harold Thimbleby, Peter Wallis, and Sean Zdenek.

\section{References}

Aldis, O., 1975. Playfighting. Academic Press, New York, NY.

Bickmore, T.W., Picard, R.W., 2005. Establishing and maintaining longterm human-computer relationships. ACM Transactions on Computer-Human Interaction 12, 293-327.

Billerter, J., 1997. Look at what happens to telltales and buffaloes. In: AAAI Fall Symposium on Socially Intelligent Agents, Cambridge, MA, pp. 7-9.

Cassell, J., 2000. Embodied conversational interface agents. Communications of the ACM 43, 70-78.

De Angeli, A., Brahnam, S., Wallis, P., 2005. ABUSE: the dark side of human-computer interaction. In: Buono, P., Costabile, M.F., Paterno, F., Santoro, C. (Eds.), Interact 2005 Adjunct Proceedings, Rome, pp. 91-92.

De Angeli, A., Brahnam, S., Wallis, P., Dix, A., 2006. Misuse and abuse of interactive technologies. In: CHI '06 Extended Abstracts on Human Factors in Computing Systems, Montreal, April 22, pp. 1647-1650.

Dunne, A., Rabby, F., 2001. Design Noir: The Secret Life of Electronic Objects. Birkhäuser, Basel, Switzerland.

Fiske, S.T., Taylor, S., 1991. Social Cognition. McGraw-Hill, New York.

Foucault, B., Mentis, H.M., Sengers, P., Welles, D., 2007. Provoking sociability. In: Press, A. (Ed.), CHI Adjunct Proceedings.

Lorenz, K.Z., 1939. Comparative study of behaviour. Verhandlungen der Deutschen Zoologischen Gesellschaft 12, 69-102.

Nathan, L.P., Klasnja, P.V., Friedman, B., 2007. Value scenarios: A technique for envisioning systemic effects of new technologies. In: CHI 2007. 
Reeves, B., Nass, C.I., 1996. The Media Equation: How People Treat Computers, Television, and New Media like Real People and Places. CSLI Publications and Cambridge University Press, Stanford, CA.

Rickenberg, R., Reeves, B., 2000. The effects of animated characters on anxiety, task performance, and evaluations of user interfaces. In: SIGCHI Conference on Human Factors in Computing Systems, The Hague, The Netherlands, pp. 49-56.

Turkle, S., 1997. Life on the Screen. Touchstone, New York.

Yee, N., Bailenson, J.N., Rickertsen, K., 2007. A meta-analysis of the impact of the inclusion and realism of human-like faces on user experiences in interfaces. In: Conference on Human Factors in Computing Systems CHI 2007 San Jose, CA, USA, pp. 1-10.
Sheryl Brahnam

College of Business Administration, Computer Information Systems, Missouri State University, 901 S. National, Springfield, MO 65804, USA E-mail address: sbrahnam@missouristate.edu

Antonella De Angeli *

Manchester Business School, The University of Manchester, Booth Street West, Manchester M15 6PB, UK E-mail address: Antonella.de-angeli@manchester.ac.uk 